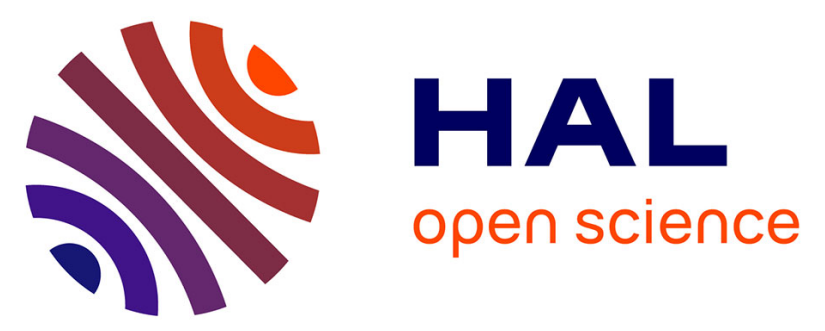

\title{
Quantitative Analysis of grafted CNT dispersion and of their stiffening of polyurethane (PU)
}

\author{
M. H. Jomaa, Lucian Roiban, D. S. Dhungana, J. Xiao, J.Y. Cavaillé, L. \\ Seveyrat, L Lebrun, G. Diguet, Karine Masenelli-Varlot
}

\section{- To cite this version:}

M. H. Jomaa, Lucian Roiban, D. S. Dhungana, J. Xiao, J.Y. Cavaillé, et al.. Quantitative Analysis of grafted CNT dispersion and of their stiffening of polyurethane (PU). Composites Science and Technology, 2019, 171, pp.103-110. 10.1016/j.compscitech.2018.12.012 . hal-02156618

\section{HAL Id: hal-02156618 https://hal.science/hal-02156618}

Submitted on 14 Jun 2019

HAL is a multi-disciplinary open access archive for the deposit and dissemination of scientific research documents, whether they are published or not. The documents may come from teaching and research institutions in France or abroad, or from public or private research centers.
L'archive ouverte pluridisciplinaire HAL, est destinée au dépôt et à la diffusion de documents scientifiques de niveau recherche, publiés ou non, émanant des établissements d'enseignement et de recherche français ou étrangers, des laboratoires publics ou privés. 


\section{Quantitative Analysis of grafted CNT dispersion and of their stiffening of polyurethane (PU)}

M. H. Jomaa ${ }^{\mathrm{a}, \mathrm{d}}$, L. Roiban ${ }^{\mathrm{a}}$, D. S. Dhungana ${ }^{\mathrm{a}, \mathrm{e}}$, J. Xiao $^{\mathrm{a}}$, J.Y. Cavailléa,b, L. Seveyrat ${ }^{\mathrm{c}}$, L. Lebrun $^{\mathrm{c}}$, G. Diguet ${ }^{\mathrm{b}}$, K. Masenelli-Varlot ${ }^{\mathrm{a}}$.

${ }^{a}$ Univ. Lyon, INSA-Lyon, Université Claude Bernard Lyon 1, MATEIS, UMR 5510, CNRS, 7 avenue Jean Capelle, F-69621 Villeurbanne Cedex, France

${ }^{b}$ ELyTMaX, UMI 3757, CNRS, Tohoku University and Université de Lyon, International Joint Unit, Tohoku University, Room\#503, MaSC Building, Katahira 2-1-1, Aoba-Ku, Sendai, 9808577, Japan

${ }^{c}$ Univ Lyon, INSA-Lyon, LGEF, F-69621 Villeurbanne cedex, France

${ }^{d}$ Current location: Queen's University Belfast, Queen's Advanced Micro Engineering Centre, Belfast, BT9 5AH, UK

${ }^{e}$ Current location: CNRS, CNRS-LAAS, University of Toulouse, 7 Avenue du Colonel Roche, F-31400 Toulouse, France

* Corresponding authors: MATEIS, bâtiment B. Pascal, 7 avenue J. Capelle, 69621 Villeurbanne cedex, France. Lucian.Roiban@insa-lyon.fr ; Karine.Masenelli-Varlot@insalyon.fr

\footnotetext{
Abstract

Electroactive devices are developed for energy conversion purposes. In particular, polyurethanes (PU) are lightweight and flexible materials, which have demonstrated their ability to convert electrical energy into mechanical energy (actuation by electrostriction) and vice-versa (energy harvesting). It has been shown that energy conversion efficiency can be increased by incorporating carbon nanotubes (CNTs) into a PU matrix. The counterpart of this
} 
improvement is the stiffness increase, which in turn limits the electrostriction efficiency. On the other hand, it is well known that CNTs are hardly dispersed in a polymeric matrix, and that the interfacial adhesion strength is generally poor. One solution to improve both dispersion and adhesion consists in grafting polymeric chains onto the CNT surfaces. As most of the works dedicated to improve material electroactivity are mainly empirical, this work aims to (i) better characterize these material microstructures by electron tomography, through the measurement of the CNT tortuosity, the CNT-CNT minimum distance and the number of their contacts, and (ii) and to predict their mechanical stiffness from these microstructural data. From electron microscopy observations of the studied materials, CNTs can be assumed to be composed of successive stiff rods of measured length and orientation, linked together by flexible kinks. Their mechanical stiffening effect in PU is, simply and in an original way, evaluated using the classical analytical equations derived by Halpin and Kardos, accounting for the microstructural parameters determined by electron tomography. It appears clearly that, due to their tortuosity and despite their ultra-high longitudinal stiffness, CNTs only poorly stiffen soft matrices. Fully stretching $10 \mu \mathrm{m}$ long nanotubes increases the composite modulus by almost 10 for a fraction of only 2 vol. $\%$.

Keywords: carbon nanotubes; polymers; smart materials; scanning/transmission electron microscopy (STEM); mechanical properties

\section{Introduction}

The last developments in electroactive materials (EAP) show promising properties especially for actuation and energy harvesting applications. Compared to inorganic materials, these versatile polymers have various attractive properties, such as being lightweight, inexpensive and easy to manufacture. A tremendous amount of research and development has proved that EAPs can change their size or their shape when stimulated by an external electric field, meaning 
they can convert electrical energy into mechanical energy. Among the various EAPs, polyurethane (PU) elastomers are of great interest due to their significant electroactivity [1] combined with flexibility, high mechanical strength and biocompatibility with blood and tissues $[2,3]$. In addition, it has been shown that the incorporation of nanofillers, such as carbon nanotubes (CNTs), into PU matrices can also enhance their electromechanical properties [4]. However, CNTs are difficult to disperse in a polymeric matrix and it is worthy to notice that the CNT-matrix interfacial adhesion strength is generally poor [5]. Furthermore, in composite materials and especially in nanocomposites, the improvement of the materials electromechanical performance is generally related to (i) the dispersion, (ii) the interaction of nanotubes with the host polymer (interfacial adhesion strength), and (iii) the alignment and orientation of the nanotubes in the polymer matrix [6]. One solution to improve both dispersion and adhesion consists in functionalizing CNTs by grafting polymer chains onto their surfaces $[7,8]$.

In order to highlight the effect of grafting on the dispersion of CNTs in polyurethanes, several parameters have to be precisely determined, such as the distance between CNTs, the number of the contacts between them, their orientation, their curvature as well as the distance between entanglements/contacts. Measuring such parameters requires a three-dimensional characterization. Electron tomography is undoubtedly the most pertinent technique able to perform a three-dimensional analysis of polymer/CNT microstructures. Few electron tomography studies dealt with the characterization of polymer / CNT nanocomposites $[9,10,11$, 12, 13]. To our knowledge, only Natarajan et al. [14] reported a quantitative analysis of the tomograms and related the evolution of several morphological parameters with the CNT volume fraction in epoxy-based nanocomposites. Alternatively, 2D images have been used to quantify the CNT orientation $[15,16]$ or the mean distance between neighboring CNTs [17]. Although 
they could provide nice agreements with experimental data, the methods used could contain artifacts due to the use of $2 \mathrm{D}$ projections of a 3D volume.

The aim of this work is to analyze the effect of CNTs and their grafting onto the actuation properties of PU. For this purpose, we first quantify several structural parameters. In a second step, we predict their mechanical stiffness from the microstructural data obtained by electron tomography. It appears clearly that, due to their tortuosity and despite their ultra-high longitudinal stiffness, CNTs only poorly stiffen soft matrices.

\section{Materials and methods}

Films composed of a polyether-based thermoplastic polyurethane (Estane 58888 NAT021 Lubrizol) and either grafted or non grafted multi-walled carbon nanotubes (CNT) (from Cheap Tubes Inc.) were prepared by solution casting according to a procedure described elsewhere [18]. Briefly, CNTs had an average diameter of $30 \mathrm{~nm}$ and lengths ranging between 10 and 20 $\mu \mathrm{m}$. A "grafting onto" technique was used to graft PU chains onto CNTs [19]. Figure 1(a) and (b) show typical Transmission Electron Microscopy (TEM) images of as-received CNTs and grafted CNTs, respectively.

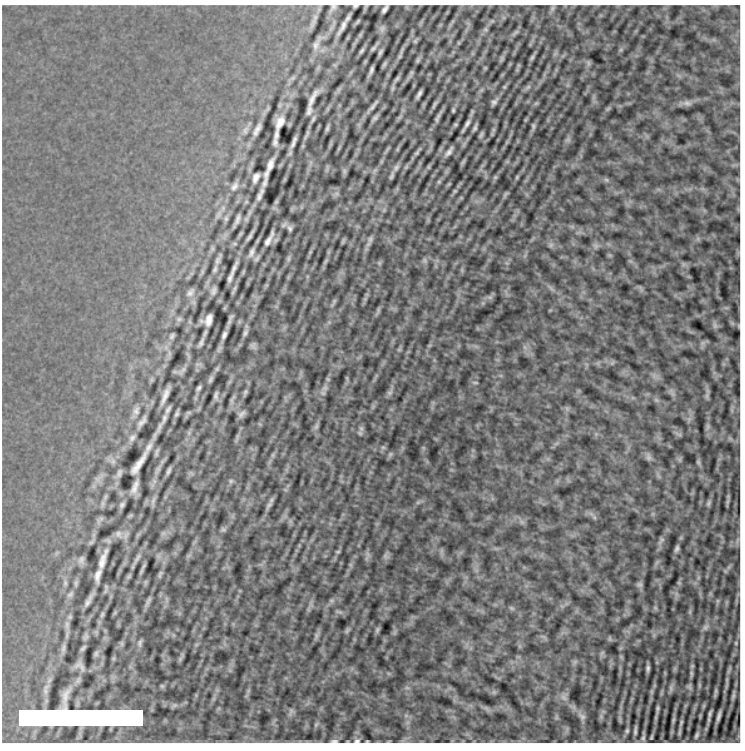

a)

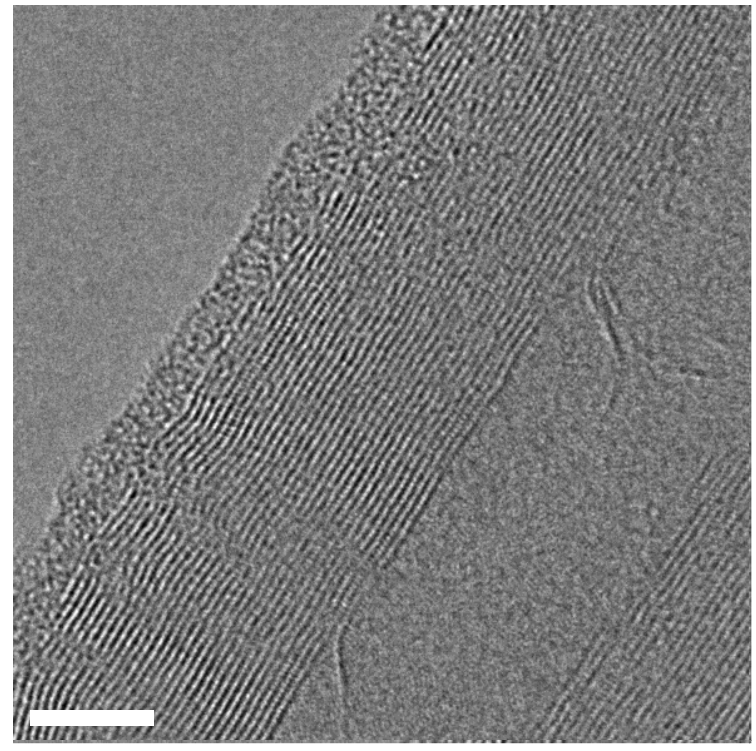

b) 
Figure 1: TEM images of the CNTs a) before any treatment and b) with PU chains grafted onto the sidewalls, forming a thin amorphous layer.

The polymer films were prepared by a solution casting method. Before use, PU granules were heated at $350 \mathrm{~K}$ for 3 hours. The grafted CNTs were first dispersed in N,N-dimethylformamide (DMF, Sigma-Aldrich D158550, $99 \%$ ) using sonication in an ultrasonic bath (Bioblock Scientific TS540 (power $10 \%$ )) and /or using an ultrasonic processor with a $7 \mathrm{~mm}$ sonotrode (Hielscher UP400S $400 \mathrm{~W}, 24 \mathrm{kHz}$, (power $30 \%$ )). PU granules were added to this solution with a ratio of 15 wt. $\%$ of PU into DMF. The solution was heated at $350 \mathrm{~K}$ for 4 hours under mechanical agitation, until a homogeneous solution was obtained. This operation was carried out in a closed device, to avoid evaporation of DMF and to ensure good reproducibility of films. Then, the solution was kept overnight to remove air bubbles. Afterwards, this solution was cast on glass plates with an Elcometer 3700 Doctor Blade ${ }^{\circledR}$ film applicator, put in an oven at 335 $\mathrm{K}$ for one day, and then removed from the glass. A second heating treatment at a temperature below the HS melting temperature was performed at $400 \mathrm{~K}$ for $3 \mathrm{~h}$ in order to eliminate any residual solvent. The final thickness of the films was measured to be about $100 \mu \mathrm{m}$ after drying. In the following, the blade displacement will be referred as "Machine direction" or Md. The normal direction $(\mathrm{Nd})$ is perpendicular to the film, while the transverse one $(\mathrm{Td})$ is in the film, perpendicular to Md. The directions are schematically represented in Figure 2 (a).

Two samples composed of PU with 2 wt.\% of either grafted or ungrafted CNTs were prepared for electron tomography analysis. The specimens were embedded in epoxy and microtomed at low temperature $(\mathrm{T}=200 \mathrm{~K})$ with a Reichert Ultracut $\mathrm{S}$ cryo-ultramicrotome equipped with a $35^{\circ}$ diamond knife (knife speed set to $1 \mathrm{~mm} / \mathrm{s}$, knife movement $100 \mathrm{~nm}$ ). The cutting was perpendicular to $\mathrm{Md}$, i.e. in the $(\mathrm{Nd}, \mathrm{Td})$ plan, along the $\mathrm{Nd}$ direction; Figure 2 (b) gives a schematic representation of the film orientation during ultramicrotomy. Ultrathin sections were 
deposited on a copper grid covered with a holey carbon film. Gold nanoparticles of a diameter of $15 \mathrm{~nm}$ were deposited on the surface of the thin sections. They were used to enhance the contrast during acquisition and to facilitate the alignment of the projections after the tilt series acquisition.

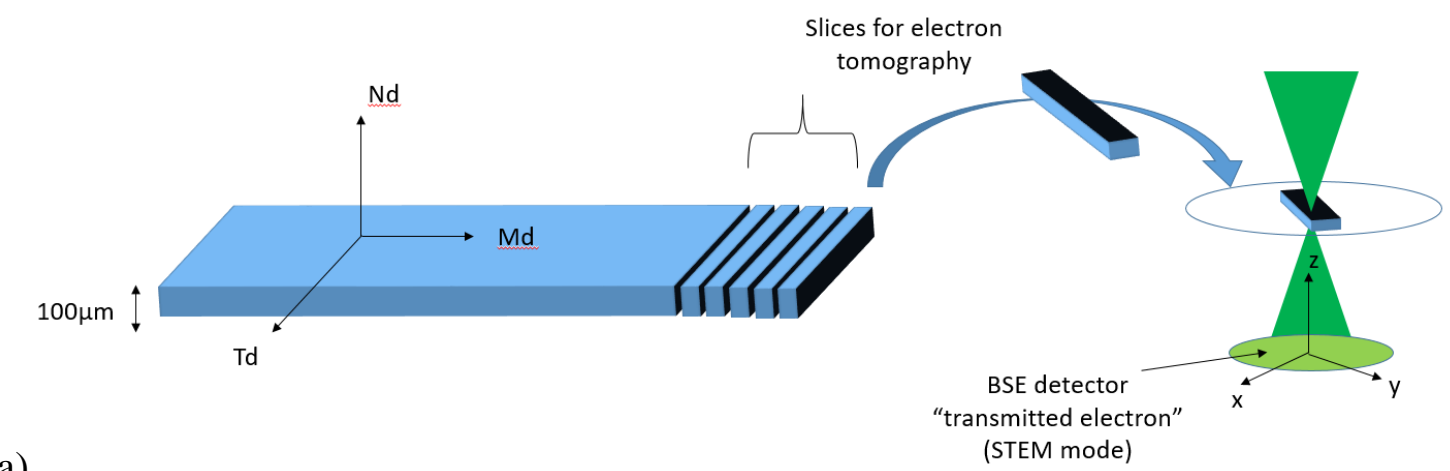

a)

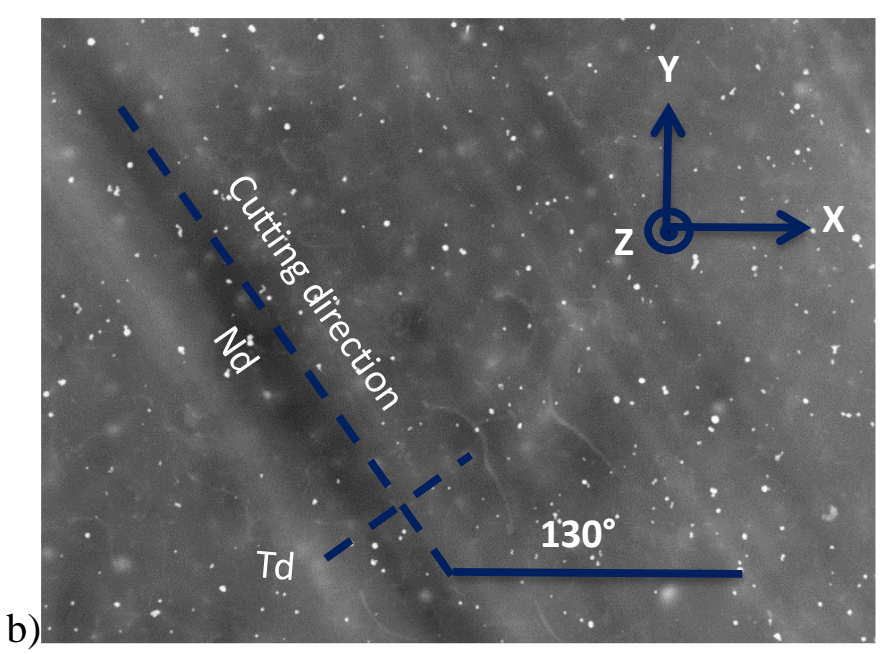

Figure 2: Definition of the sample orientation and corresponding frames. a) Schematic representation of the elaboration process with the directions $\mathrm{Md}, \mathrm{Td}$ and $\mathrm{Nd}$. A schematic representation of the electron tomography set-up for environmental SEM is given with the tomography referential $(\mathrm{x}, \mathrm{y}, \mathrm{z})$ showing that $\mathrm{z}$-axis is the Md direction. b) Low resolution image of an ultramicrotomy thin section with the $\mathrm{Nd}$ and $\mathrm{Td}$ directions reported to the $\mathrm{x}, \mathrm{y}, \mathrm{z}$ directions used in electron tomography. The white spots are Au nanoparticles.

Tilt series were acquired with a FEI XL-30 FEG environmental scanning electron microscope (ESEM) operating at $30 \mathrm{kV}$ under $400 \mathrm{~Pa}$ of water partial pressure. The environmental mode 
allows a gas flow having the capacity to drive out the charges, thus facilitating the study of nonconductive samples. The sample was placed on a home-made holder allowing a high tilt angle [20]. A solid detector placed $10 \mathrm{~mm}$ below the sample was used to collect the electrons scattered between $14^{\circ}$ and $40^{\circ}$. The contrast obtained in the recorded images is mass-dependent [21]. The ESEM performances combined with the chosen imaging mode push the resolution down to a few nm [22], including in three dimensions [20]. Tilt series were acquired with angles ranging from $+66^{\circ}$ to $-66^{\circ}$, with a constant tilt step of $2^{\circ}$. Prior to the acquisition, the stage and the samples were cleaned in situ using an Evactron system to avoid contamination during the tilt series acquisition. Moreover, the region of interest was irradiated during 5 minutes with the electron beam to avoid shrinkage problems during acquisition. Although this is known to induce chemical changes inside the polymeric phase, it is thought not to affect the CNT distribution. In electron tomography, the axes $(\mathrm{x}, \mathrm{y}, \mathrm{z})$ are defined, where $\mathrm{z}$ represents the direction of the sample depth, $\mathrm{x}$ and $\mathrm{y}$ being the horizontal and vertical axes in the image. From the geometry used in cryo-ultramicrotomy, it is obvious that z corresponds to Md. The relation between (x, y) and (Md, Td) is given by linear defects in the sample, visible at low magnification (see Figure 1 (b)). These defects can be attributed to scratches introduced by the diamond knife during cryoultramicrotomy. Therefore, the linear defects correspond to the cutting direction, $\mathrm{Nd}$. In the film containing grafted CNTs, the angle between $\mathrm{Nd}$ and $\mathrm{x}$ is measured to be equal to $+130^{\circ}$ (or $310^{\circ}$ ) and the angle between $\mathrm{Td}$ and $\mathrm{x}$ is thus equal to $40^{\circ}$ (or $220^{\circ}$ ).

After the tilt series acquisition, an accurate alignment of the projections is performed in ETOMO, using gold nanoparticles as fiducial markers [23]. The volume reconstruction is done using the TomoJ [24] plugin of ImageJ software, with the ART algorithm (15 iterations). The ART reconstruction algorithm is an iterative algorithm that projects the reconstructed volume and compares one by one the calculated projections with the original ones. The difference between projections is projected until both a chosen number of iterations and convergence are 
reached [25]. Other reconstruction algorithms can be found in the literature and have been compared in the literature [26], [27], [28]. In the present study, we preferred to use ART, as it allows a better preservation of the reconstructed object shape after correction by the elongation factor. However, segmentation is more time-consuming with ART as the reconstructed volume is noisy. Segmentation is carried out combining Trainable Waka Segmentation [29], with other plug-ins [30] in Fiji, and 3D Slicer (http://www.slicer.org/) [31], further used for advanced segmentation.

\section{Results and discussion}

Figure 3 a) and b) show orthogonal cross-sections extracted from the reconstructed volumes, in the case of nanocomposites containing grafted and ungrafted CNTs, respectively. The white dots are the gold nanoparticles. The contrast allows identifying the CNT distribution within the PU matrix. From the volume analysis at different depths and orientations, the thicknesses of the analyzed samples are measured to be around $350 \mathrm{~nm}$ and $450 \mathrm{~nm}$ for the nanocomposites containing grafted and ungrafted CNTs, respectively. The dispersion of grafted CNTs is found to be rather uniform and only small heterogeneities are observed within the analyzed volume, whereas in the sample containing ungrafted CNTs, regions with different densities of CNT are observed. Grafting CNTs seems to improve the CNT dispersion within the PU matrix. This is consistent with the impressive improvement of the stability of the CNT suspension in DMF upon grafting [18]. The interfacial adhesion strength is also expected to be excellent since the same chemical component is present in both sides of the interfaces (PU in the grafted layer and in the matrix). 


\subsection{Quantification and control of the CNT dispersion state}

The volume quantification is carried out considering two main objectives: (i) characterization of the CNT dispersion state and (ii) measurement of the CNT orientation state. To facilitate these objectives, virtual markers are numerically placed in the three-dimensional model obtained by segmentation of the reconstructed volume. The positions of the markers are considered in order to obtain clear statistical information of possible contacts between the CNTs within the sample.

Each CNT being considered as a polyline, the virtual markers are placed manually at the endpoints where a change in the direction of the CNT is observed. Then, the spherical coordinates of the virtual markers are extracted (see Figure 4 a)). Two consecutive markers define a segment and one CNT is composed of a variable number of segments having different orientations. From the markers coordinates, each CNT segment is then characterized by its length $r$, polar angle $\theta$ (representing the segment orientation with respect to the $\mathrm{z}$ axis), and azimuthal angle $\varphi$ (representing the segment orientation in the $\mathrm{x}, \mathrm{y}$ plane, with respect to the $\mathrm{x}$ axis). Analyzing the segment positions and orientations give rise to a direct measurement of the CNT dispersion and orientation within the PU matrix.

a)

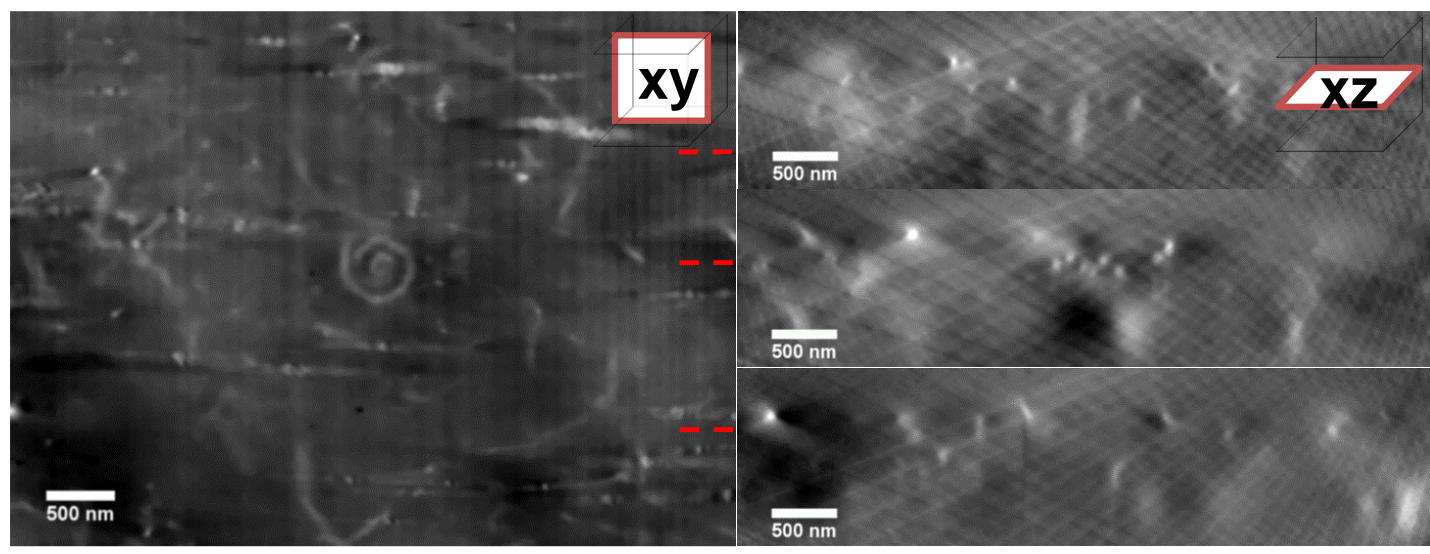


b)

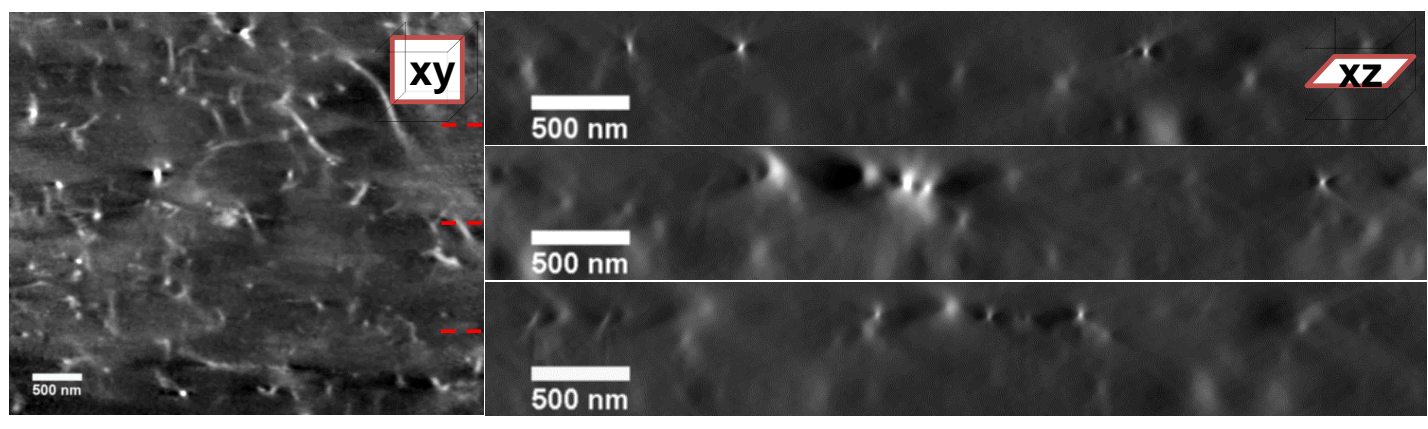

c)
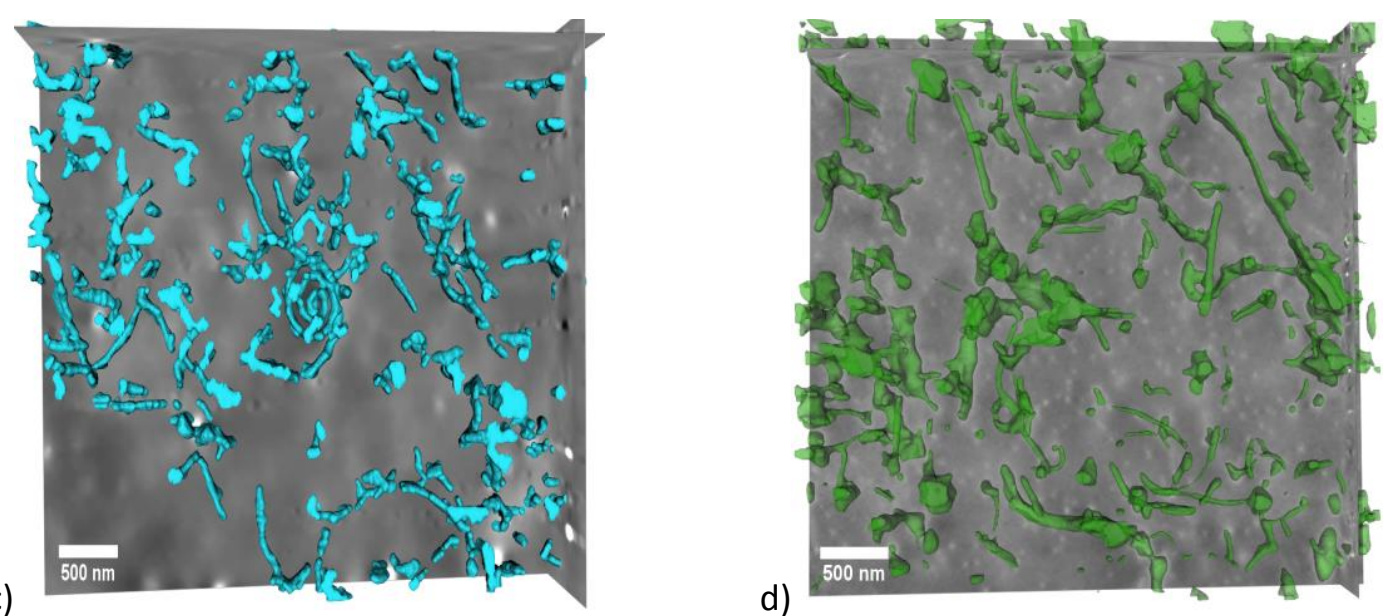

Figure 3: Orthogonal cross-sections through the reconstructed volumes of the samples:

a) with grafted CNTs, b) with ungrafted CNTs. The red dashed lines show the intersection between the orthogonal slices. c) and d) three-dimensional models obtained after segmentation, with grafted CNTs in blue and ungrafted CNTs in green.

a)

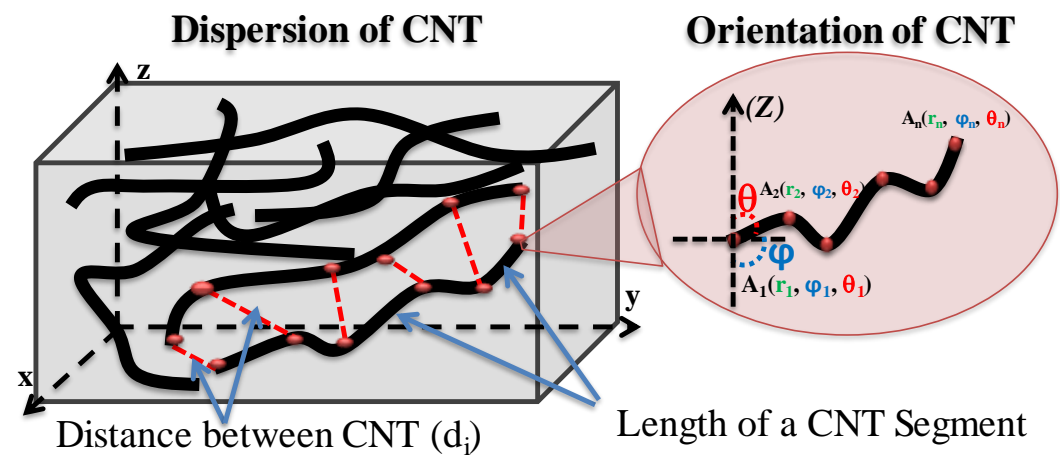


b)

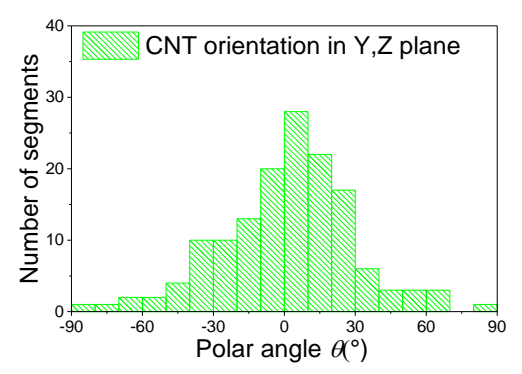

c)

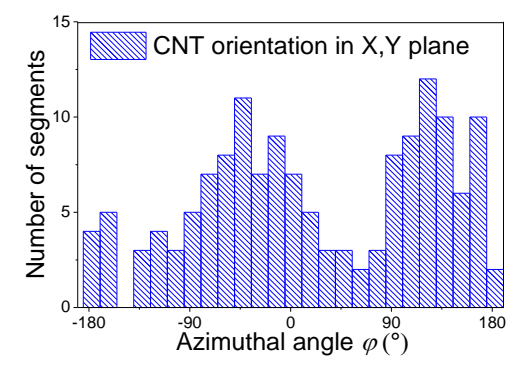

Figure 4: Characterization of the CNT dispersion and orientation: a) Schematic representation of the quantification method used for the analysis of the CNT orientation. b) CNT orientation for the grafted sample (with $2 \%$ of CNTs), the polar angle $\theta$ representing the orientation of each segment in the YZ plane. c) CNT orientation for the grafted sample, the azimuthal angle $\varphi$ representing the orientation of each segment in the x,y plane.

The quantitative parameters describing the CNT dispersion state are shown in Table 1 . The minimum distances between CNTs are measured using the segment method applied on the three-dimensional model. The average length of the segments slightly increases from ungrafted to grafted containing 2 vol. $\%$ of CNTs suggesting that the grafted molecules help to rigidify the CNTs. The further increase, observed from 2 vol.\% to 4 vol.\% of grafted CNTs, might rather be a dispersion-related phenomenon, such as bundles formation, for instance. The values obtained confirm that grafted CNTs are not in direct contact with each other in the nanocomposites containing 2 vol.\% of grafted CNTs and a minimum distance around $50 \mathrm{~nm}$ is obtained. On the contrary with 2 vol. $\%$ of ungrafted CNTs and 4 vol. $\%$ of grafted CNTs, 11 and 5 contacts between CNTs have been found within the analyzed volumes, respectively. The formation of percolating paths may reflect overall conductive materials. This suggests that for 2 vol.\% of CNTs, grafted CNTs are better dispersed than ungrafted CNTs. Moreover, the percolation threshold seems to be close to 4 vol.\% of grafted CNTs. This is in very good agreement with macroscopic electrical measurements, where the percolation threshold was 
found to lie between 4 and 5 vol.\% on the same films [18]. In the following, only grafted CNTs will be considered.

Table 1: CNT distribution within the volume of PU: analyzed volume, number of contacts between the CNTs counted in the total analyzed volume, and minimum distance between CNTs reported to the matrix volume. The mean length of the CNT segments is also given.

\begin{tabular}{ccccc}
\hline Type of & $\begin{array}{c}\text { Mean length } \\
\text { of CNT }\end{array}$ & $\begin{array}{c}\text { Number of } \\
\text { Composite }\end{array}$ & Minimum & Analyzed \\
& segments & & Distance & volume \\
\hline Ungrafted & & 11 & 0 & $7.520 \mu \mathrm{m}^{3}$ \\
CNTs & $500 \mathrm{~nm}$ & & & \\
$(\mathbf{2}$ vol.\%) & & 0 & & $7.550 \mu \mathrm{m}^{3}$ \\
\hline Grafted CNTs & $590 \mathrm{~nm}$ & & & \\
$(\mathbf{2}$ vol.\% $)$ & & 5 & 0 & $6.600 \mu \mathrm{m}^{3}$ \\
\hline Grafted CNTs & $800 \mathrm{~nm}$ & & & \\
$(\mathbf{4}$ vol.\% $)$ & & & & \\
\hline
\end{tabular}

For PU grafted with $2 \%$ of CNTs, the polar angles $\theta$ follow a Gaussian distribution centered near $\theta \sim 0^{\circ}$ (Figure $4 \mathrm{~b}$ )), i.e. the Md direction. The azimuthal angle distribution is bimodal, with two preferential orientations at $\varphi \approx 130^{\circ}$ and $310^{\circ}$ (Figure 4 (c)). These supplementary angles actually represent the same CNT planar direction and this was previously identified to the cutting direction for cryo-ultramicrotomy, hence it corresponds to the direction $\mathrm{Nd}$. The preferential values of $\theta$ and $\varphi$ clearly indicate that the CNTs were in the film plane. Moreover, the CNT orientation state in the (Md, Td) place can be deduced from the values of $\theta$ and $\varphi$. When projecting a CNT segment onto the $(\mathrm{Md}, \mathrm{Td})$ plane, it is possible to define an angle in 
this plane, $\beta$, between the projected segment and the Md axis. Using geometrical considerations, it can be shown that

$$
\tan (\beta)=\tan (\theta) \cdot \cos \left(\varphi-40^{\circ}\right)
$$

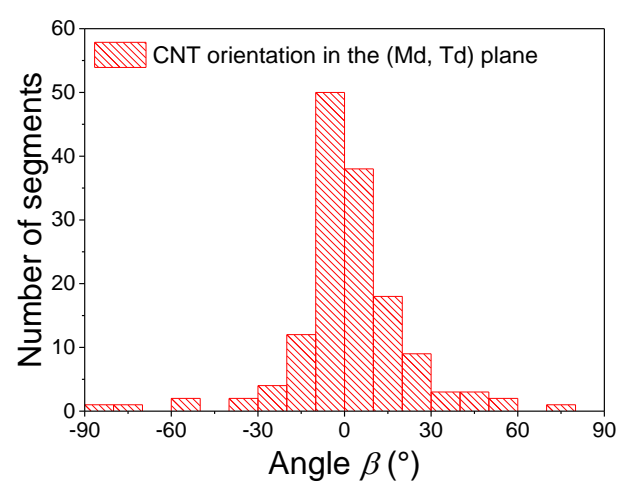

Figure 5: CNT orientation in the (Md, Td) plane, for the sample containing 2 vol.\% of grafted CNTs. The angle $\beta$ represents the angle between a CNT segment and the Md axis.

The distribution of angles $\beta$ indicates that the CNTs are rather well oriented along the $\mathrm{Md}$ direction (see Figure 5). The appearance of a preferential orientation has already been observed in the literature. For example, Ma et al. [32] used an electric field to align pristine or functionalized MWCNTs. Xie et al. [33] reviewed studies related to alignment of CNTs in a matrix, especially using force and magnetic fields, electrospinning and procedures involving liquid crystals. More recently, K. Gnanasekaran et al. [15] quantified TEM images of spincoated latex/CNT composite films and could evidence CNT alignment along the spin-coating direction, providing that the measurements are carried out on large enough areas. In our case, the film was cast on glass using a Doctor Blade Film Applicator. This technique does not give rise to intense shear and no external force or electric field is applied but an anisotropic orientation state is already obtained. 


\subsection{Evaluation of mechanical stiffening of PU by CNTs}

The main purpose of this section is to evaluate if it is possible to relate the parameters found from the 3D analysis, presented above, with the stiffening observed by mechanical measurements. There are few cases where the problem of stiffening effect can be evaluated with analytical equations as, in most cases, an accurate evaluation requires the use of numerical simulations often based on finite element calculations. The rare cases where analytical equations can be powerful and lead to rather accurate predictions concern (i) spherical or ellipsoidal particles $[34,35]$ and (ii) short stiff particles or rods. For instance, Halpin and Tsai [36] and Halpin and Kardos [37] proposed equations where not only the shape factor of the particles (i.e. their length divided by their diameter) but also their elastic constant anisotropy might be considered.

However, for the case where particles are in fact flexible short fibers, like CNTs, it is extremely difficult to model the composite mechanical properties. J. Pascual et al. [38] found discrepancies between experimental elastic modulus values and the Halpin-Tsai model. They attributed the differences to the presence of CNT aggregates but the CNT waviness was not discussed. Yet, it is well known that the curvature (bending) of fibers decreases drastically their stiffening effect [39], compared to straight fibers especially when the stress is mainly applied parallel to the fiber axis. The effect of the CNT waviness on the thermal properties of polycarbonate/CNT composites has, for instance, been evidenced and modeled by Yu et al. [40]. The model was also modified to take into account randomly oriented fillers [41] or by introducing a "waviness ratio" [42, 43] or an "orientation factor" [44], both parameters being unfortunately not experimentally measurable.

As there is no way to model the exact microstructure of these nanocomposites, and in order to relate the stiffening induced by the nanotubes to their parameters given in Table 1, we assume in the following that CNTs consist in a series of stiff rods linked together by flexible joints. As 
the CNT flexibility is very high compared to their longitudinal compliance and again because there is no way to go deeper in this analysis, it will be assumed that the main stiffening effect comes from the stress transfer from the matrix to the CNT segments. This means that the knees (junctions between two segments) are assumed to transmit no moment from a segment to the adjacent one. From previous studies on composite materials stiffened by perfectly straight cellulose nanocrystals [45], the effect of the stiffness of contacts between the cellulose rods was quantified. Depending on the process, these contacts are very strong when hydrogen bonds are formed between nanocrystals, or very weak if hydrogen bonds are not allowed to form [46]. The composite stiffness differs by almost 3 decades for 5 vol.\% of cellulose whiskers if contacts are stiff or not. If we agree that the knees play almost no role, the situation is close to the one where straight segments are randomly dispersed in the matrix. In the present case, such assumption gives a unique opportunity to evaluate the effect of CNTs on the mechanical properties of the composites with a model fully based on the composite structure and on the characteristics of each component, i.e. without any adjustable parameter.

As a first attempt, we consider in the following that in the materials studied here, each CNT can be considered as a set of stiff straight rods independent to each other, as far as deformation is very small. The main stiffening mechanism consists in stress transfer from the matrix towards these rods, while the flexible joints play almost no role.

The components of the elastic constant tensor can be written as:

$$
\frac{E_{i i}}{G_{m}}=\frac{2 E_{f i i}\left(1+{ }_{m}\right)\left(1+{ }_{i i} X_{f}\right)+4_{i i}\left(1+{ }_{m}\right)^{2}\left(1 X_{f}\right) G_{m}}{E_{f i i}\left(1 X_{f}\right)+2\left({ }_{i i}+X_{f}\right)\left(1+{ }_{m}\right) G_{m}}
$$

where $E_{i i}$ refers to the longitudinal composite modulus (direction $i$ ), $E_{f i i}$ refers to the fiber longitudinal modulus, $G_{m}$ refers to the isotropic matrix shear modulus, ${ }_{m}$ to the matrix Poisson 
ratio, $X_{f}$, the fiber volume fraction and ${ }_{11}=2\left(\frac{L}{e}\right),{ }_{12}=\left(\frac{l}{e}\right)^{\sqrt{3}},{ }_{22}=2\left(\frac{l}{e}\right)$, with $L, l$ and $e$, the length, thickness and width of the rods.

The composite shear modulus $G$ is given by:

$$
G=\frac{E_{11}+E_{22}\left(\begin{array}{ll}
1 & 12
\end{array}\right)}{8\left(\begin{array}{lll}
1 & 12
\end{array}\right)}+\frac{G_{12}}{2}
$$

where ${ }_{12}=X_{f}+\left(\begin{array}{ll}1 & X_{f}\end{array}\right)_{m}$, and ${ }_{12}={ }_{21}$

and,

$$
\frac{G_{12}}{G_{m}}=\frac{G_{f}\left(1+{ }_{12} X_{f}\right)+{ }_{12}\left(1 \quad X_{f}\right) G_{m}}{G_{f}\left(1 \quad X_{f}\right)+\left({ }_{i i}+X_{f}\right) G_{m}}
$$

From Equ.2, using the complex expression of the mechanical moduli to account for the matrix viscoelasticity $\left(G_{m}=G_{m}^{\prime}+i G_{m}^{\prime \prime}\right)$, it is then possible to calculate the composite shear modulus, in which $G=G^{\prime}+i G^{\prime \prime}$. Within the temperature range considered in this study, the CNT elastic constants (here $G_{f}$ ) remain almost temperature-independent so that the composite shear modulus can be calculated for all temperatures using for the matrix modulus $G_{m}$, available from experimental measurements. In Figure 6, the real part of the composite shear modulus $G^{\prime}$ is plotted against temperature at constant frequency of $0.1 \mathrm{~Hz}$; the measurements are made with an inversed torsion modulus described in literature [47]. As the stiffening effect is mainly effective when the matrix is soft, the shear modulus is normalized at $1 \mathrm{GPa}$ at $150 \mathrm{~K}$. The main reason is that torsional measurements are very accurate to detect relative changes. However, they cannot really provide absolute values: the measured modulus requires the knowledge of a geometrical parameter, which depends on the cube of the sample thickness, leading to a large uncertainty just because of sample dimension irregularity due to their preparation. 


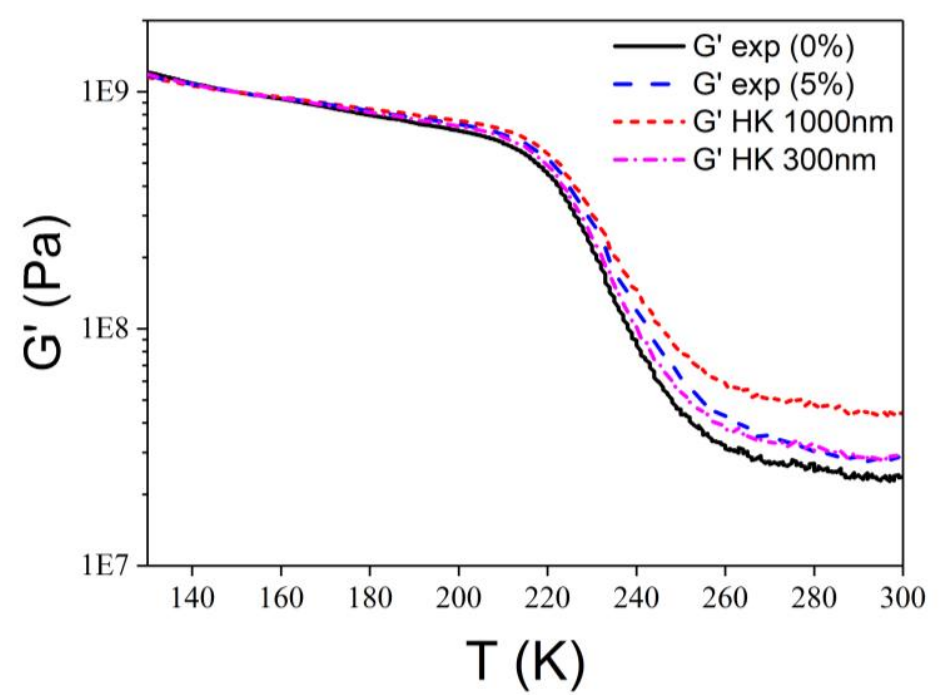

Figure 6: Storage shear modulus $G^{\prime}$ (in $\mathrm{Pa}$ ) versus temperature $T$ (in $\mathrm{K}$ ), for the pure matrix and 5 vol.\% CNT, at $0.1 \mathrm{~Hz}$ (curves "G' pure" and "G'exp (5\%)", respectively). Two calculated curves ( $G^{\prime}$ HK $300 \mathrm{~nm}$ and $G^{\prime}$ HK $1000 \mathrm{~nm}$ ) obtained using the HK equation with rods of $300 \mathrm{~nm}$ and $1000 \mathrm{~nm}$ in length, respectively, are also displayed.

Calculated curves are also shown in Figure 6. They were obtained using the HK equation, on the basis of the following statements:

(i) CNTs are assumed to be purely elastic (no viscous components) with elastic constants independent from temperature;

(ii) The HK equation is accounting for the complex modulus of the matrix, even though for sake of simplicity, only the storage modulus is displayed;

(iii) To obtain such calculated curves, the HK equation provides the complex modulus from every experimental value of the pure matrix behavior obtained at increasing temperatures. The choice of the various CNT elastic constants comes from data in the literature, but it is worthy to notice that because CNTs are much stiffer than the matrix (especially for their longitudinal $E_{f 11}$ modulus) the result does almost not depend anymore on the exact value. Thus the chosen values were, $E_{f 11}=100 \mathrm{GPa}$, 
$E_{f 22}=10 \mathrm{GPa}, \quad m=0.49$ in the rubbery region, $f=0.3, l=e=22 \mathrm{~nm}$ (corresponding to the mean CNT diameter). $L$ is here the mean length of the CNT straight segments.

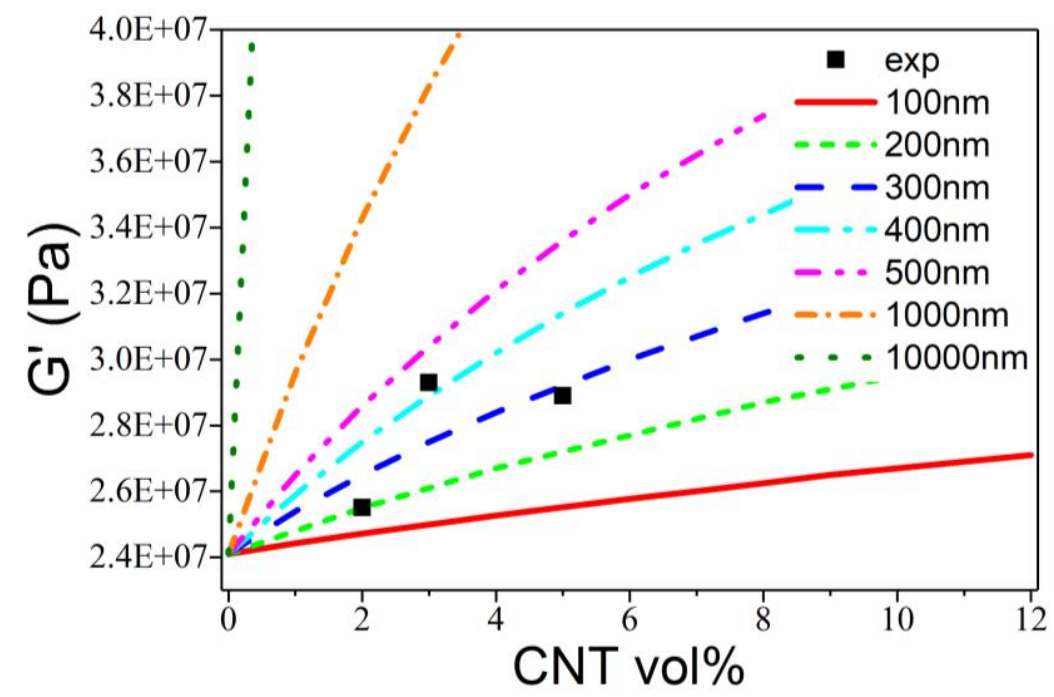

Figure 7: Composite storage modulus $\mathrm{G}^{\prime}$ in function of the CNT volume fraction, calculated with the HK equation. The black dots represent experimental data, while the curves correspond to different CNT segment lengths L.

Figure 6 shows (i) the stiffening by CNTs calculated from the HK equation, leading to values very close to experimental data (without any adjustable parameters) and (ii) the high sensitivity of $L$, as better shown in Figure 7. However, it could be noticed that the best agreement between simulations and the experiment on the composite containing 2 vol.\% of grafted CNTs is obtained for $\mathrm{L}=200 \mathrm{~nm}$, a value significantly lower than the mean segment length measured by electron tomography $(\mathrm{L}=590 \mathrm{~nm})$. This effect might be because the CNT segments are not ideally stiff. Moreover, CNT are not true rods, as assumed in the frame of the HK equation: their longitudinal tensile stiffness is extremely high compared to their compressive behavior, because of their easy buckling, which in turn decreases a lot their efficiency if their orientation is far from the macroscopic tensile direction. When taking the measured mean segment length 
( $\mathrm{L}=590 \mathrm{~nm}$ ), the calculated modulus seems to be over-estimated by less than $20 \%$. It is also noteworthy that increasing the volume fraction of CNTs from 3 to $5 \%$ does not increase the stiffness of the composite as it should be expected. In fact, for volume fractions above the percolation threshold, a certain amount of CNTs forms aggregates, which makes them less effective.

The 3D analysis was performed by electron tomography on quite large but still limited volumes (see Table 1). It is very difficult to estimate the (lack of) representativity of the 3D analysis. Nevertheless, correlating 3D analysis and mechanical behavior simulations can give interesting insights. Indeed, a good agreement has been found between simulations and experiments for the same order of magnitude of the CNT segments. This suggests that the 3D analysis is fairly representative of the whole material. However, the simulation confirms that the only way to take benefit of such stiffening fillers is to be able to stretch them. For example, considering a volume fraction of $2 \%$, the composite modulus would be of $25 \mathrm{GPa}$ with segments of $200 \mathrm{~nm}$, while it would be of $180 \mathrm{GPa}$ for segments of $10 \mu \mathrm{m}$ (full CNT length), i.e. one order of magnitude higher.

\section{Conclusions}

The aim of this paper was to evaluate the contribution of CNTs on the mechanical behavior of PU/CNT nanocomposites, as they are good candidates to design electrostrictive systems. The first part of this work dealt with the quantification of the CNT dispersion and orientation states. For this purpose, electron tomography has been performed in an Environmental Scanning Electron Microscope in the transmission mode. Electron tomograms have been reconstructed, segmented, and the CNT distribution quantified. For grafted CNTs within the analyzed volume, no contact between the CNTs was found (contrarily to what was observed with ungrafted 
CNTs). Moreover, samples containing either grafted or ungrafted CNTs exhibit similar CNT orientations: the CNTs were found to lie in the film plane and have a preferential orientation along the casting direction. The second part of the study focused onto the evaluation of mechanical properties of PU, stiffened by CNTs. A model based on analytical equations, without any adjustable parameter, was used. It is interesting to notice that, in our study, as a very rough approximation, a CNT was be assumed to be a set of separate stiff rods of which length was determined from tomography observations. The calculation results showed the consistency between microstructural observations and the stiffening effect induced by CNTs. It has been clearly shown that due to their waviness, CNT are poorly efficient as stiffening particles at the studied concentrations. Fully stretching $10 \mu \mathrm{m}$ long nanotube would however increase the composite modulus by almost 10 for a fraction of only 2 vol.\%. More generally, the method presented in this paper, namely the combination of 3D analysis and modeling, is a powerful tool to understand and predict the macroscopic properties of nanocomposites.

\section{Acknowledgements}

The authors acknowledge the French Agence Nationale de la Recherche (ANR) for financial support, under grant NAPOLECO (ANR- 2010-INTB-910-01), the Consortium Lyon SaintEtienne de Microscopie (clym.fr) for the access to the JEOL 2010F and FEI ESEM XL30-FEG microscopes, and the Centre Technologique des Microstructures for the access to the cryoultramicrotome.

\section{Conflicts of interest}

There are no conflicts of interest to declare.

\section{References}


[1] M. Watanabe, T.H., M. Suzuki, Y. Amaike Y, Electric conduction in bending electrostriction of polyurethanes, Appl. Phys. Lett. 74 (1999) 2717-2719.

[2] L. Irusta, M. J. Fernandez-Berridi, Photooxidative behavior of segmented aliphatic polyurethanes, Polym. Deg. \& Stab. 63 (1999) 113-119.

[3] C. Putson, L.L., D. Guyomar, N. Muensit, P-J. Cottinet, L. Seveyart, B. Guiffard, Effects of copper filler sized on the dielectric properties and the energy harvesting capability of nonpercolated polyurethane composites, J. Appl. Phys. 109 (2011) 024104.

[4] U. Szeluga, B. Kumanek, B. Trzebicka, Synergy in hybrid polymer/nanocarbon composites. A review, Composites Part A 73 (2015) 204-231.

[5] Z. M. Dang, L.Wang, , Y. Yin, Q. Zhang, Q.Q. Lei, Giant dielectric permittivities in functionalized carbon-nanotube/electroactive-polymer nanocomposites, Adv. Mater. 19 (2007) 852-857.

[6] Z.M. Huang, Y.Z. Zhang, M. Kotaki, S. Ramakrishna, A review on polymer nanofibers by electrospinning and their applications in nanocomposites, Composites Science and Technology 63 (2003) 2223-2253.

[7] J. Zhu, J. D. Kim, H. Peng, J. L. Margrave, V. N. Khabashesku, E. V. Barrera, Improving the dispersion and integration of single-walled carbon nanotubes in epoxy composites through functionalization, Nano Lett. 3 (2003) 1107-1113.

[8] H. Xia, M. Song, Preparation and characterization of polyurethane grafted single-walled carbon nanotubes and derived polyurethane nanocomposites, J. Mater. Chem. 16 (2006) $1843-1851$.

[9] M.H. Gass, K.K.K. Koziol, A.H. Windle, P.A. Midgley, Four-dimensional spectral tomography of carbonaceous nanocomposites, Nano Lett. 6 (2006) 376-379. 
[10] J. Yu, K. Lu, E. Sourty, N. Grossiord, C.E. Koning, J. Loos, Characterization of conductive multiwall carbon nanotube/polystyrene composites prepared by latex technology, Carbon 45 (2007) 2897-2903.

[11] H.S. Kim, J.H. Kim, C.M. Yang, S.Y. Kim, Synergistic enhancement of thermal conductivity in composites filled with expanded graphite and multi-walled carbon nanotube fillers via melt-compounding based on polymerizable low-viscosity oligomer matrix, Journal of Alloys and Compounds 690 (2017) 274-280.

[12] E.M. Campo, D. Yates, B. Berson, W. Rojas, A.D. Winter, M. Ananth, J.J. SantiagoAviles, E.M. Terentjev, Macromol. Mater. Eng. 8/2017, 1600479.

[13] F. Dalmas, L. Roiban, Three-dimensional Microstructural Characterization of Polymer Nanocomposites by Electron Tomography, in Functional and Physical Properties of Polymer Nanocomposites (eds A. Dasari and J. Njuguna), John Wiley \& Sons, Ltd, Chichester, UK (2016).

[14] B. Natarajan, N. Lachman, T. Lam, D. Jacobs, C. Long, M. Zhao, B.L. Wardle, R. Sharma and A. Liddle, The evolution of carbon nanotube network structure in unidirectional nanocomposites resolved by quantitative electron tomography, ACS Nano9 (2015) 6050-6058.

[15] K. Gnanasekaran, R. Snel, G. de With, H. Friedrich, Quantitative nanoscopy: tackling sampling limitations in (S)TEM imaging of polymers and composites, Ultramicrosc. 160 (2016) 130-139.

[16] A. Mikhalchan, T. Gspann, A. Windle, Aligned carbon nanotube-epoxy composites: the effect of nanotube organization on strength, stiffness, and toughness, J. Mater. Sci., 51 (2016) 10005-10025. 
[17] K. Gnanasekaran, G. de With, H. Friedrich, Quantitative analysis of connectivity and conductivity in mesoscale multiwalled carbon nanotube networks in polymer composites, J. Phys. Chem. C 120 (2016) 27618-27627.

[18] M. H. Jomaa, K. Masenelli-Varlot, L. Seveyrat, L. Lebrun, M.C. Dib Jawhar, E. Beyou, J. Y. Cavaillé, Investigation of elastic, electrical and electromechanical properties of polyurethane/grafted carbon nanotubes nanocomposites, Composites Science and Technology 121 (2015) 1-8.

[19] H. Xia, M. Song, J. Jin, L. Chen, Poly(propylene glycol)-Grafted Multi-Walled Carbon Nanotube Polyurethane. Macromol. Chem. Phys. 207 (2006) 1945-1952.

[20] K. Masenelli-Varlot, A. Malchère, J. Ferreira, H. Heidari Mezerji, S. Bals, C. Messaoudi, S. Marco, Wet-STEM tomography: principles, potentialities and limitations, Micros. and Microanal. 20 (2014) 366-375.

[21] Z. Saghi, P. A. Midgley, Electron tomography in the (S)TEM: from nanoscale morphological analysis to 3D atomic imaging, Annual Review of Materials Research 42 (2012) 59-79.

[22] A. Bogner, G. Thollet, D. Basset, P. H. Jouneau, C. Gauthier, Wet STEM : a new development in environmental SEM for imaging nano-objects included in a liquid phase, Ultramicroscopy 104 (2005) 290-301.

[23] M. Maiorca, E. Hanssen, E. Kazmierczak, B. Maco, M. Kudryashev, R. Hall, H. Quiney, L. Tilley, Improving the quality of electron tomography image volumes using pre-reconstruction filtering, J Struct. Biol. 180 (2012) 132-42.

[24] C. Messaoudi, T. Boudier, C.O.S. Sorzano, S. Marco, TomoJ: tomography software for three-dimensional reconstruction in transmission electron microscopy, BMC Bioinformatics 8 (2007) 288. 
[25] R. Gordon, G. Herman, Three-Dimensional Reconstruction from Projections: A Review of Algorithms, International Review of Cytology 38 (1974), 111-151.

[26 ] M. Radermacher (2007) Weighted Back-projection Methods. In: Frank J. (eds) Electron Tomography. Springer, New York, NY

[27] T. Sanders, J. D. Roehling, K. J. Batenburg, B. C. Gates, A. Katz, P. Binev, I. Arslan, Advanced 3-D Reconstruction Algorithms for Electron Tomography, Microsc. Microanal. 20 ( 2014), 794-795.

[28] D. Chen, B. Goris, F. Bleichrodt, H. H. Mezerji, S. Bals, K. J. Batenburg, G. With, H. Friedrich, The properties of SIRT, TVM, and DART for 3D imaging of tubular domains in nanocomposite thin-films and sections, Ultramicroscopy147 (2014), 137-148.

[29] I. Arganda-Carreras, V. Kaynig, C. Rueden, K. W Eliceiri, J. Schindelin, A. Cardona, H. S. Seung, Trainable Weka Segmentation: a machine learning tool for microscopy pixel classification, Bioinformatics, 33 (15) (2017), 2424-2426.

[30 ] E. H. W. Meijering, W. J. Niessen, M. A. Viergever, Quantitative Evaluation of onvolution-Based Methods for Medical Image Interpolation, Medical Image Analysis, 5 (2001), 111-12.

[31] A. Fedorov, R. Beichel, J. Kalpathy-Cramer, J. Finet, J. C. Fillion-Robin, S. Pujol, C.Bauer, D. Jennings, F. Fennessy, M. Sonka, J. Buatti, S. Aylward, J.V. Miller, S. Pieper, R. Kikinis, 3D Slicer as an Image Computing Platform for the Quantitative Imaging Network, Magn Reson Imaging. 30(9) (2010), 1323-1341.

[32] C. Ma, W. Zhang, Y. Zhu, L. Ji, R. Zhang, N. Koratkar, J. Liang, Alignment and dispersion of functionalized carbon nanotubes in polymer composites induced by an electric field, Carbon 46 (2008), 706-720.

[33] X.L. Xie, Y.W. Mai, X.P. Zhou, Dispersion and alignment of carbon nanotubes in polymer matrix : a review, Mater. Sci. Eng. R49 (2005) 89-112. 
[34] E.H. Kerner, The elastic and thermo-elastic properties of composite media, Proc. Phys. Soc. B69 (1956) 808.

[35] R. A. Dickie Interpretation of the dynamic mechanical response of heterogeneous polymer blends in terms of continuum models, Polym. Eng. Science 19 (1979) 10421045.

[36] J.C. Halpin, Environmental Factors in Composite Materials Design 1967, AFML TR 67-423.

[37] J. C. Halpin, J. L. Kardos, Moduli of crystalline polymers employing composite theory, J. Appl. Phys. 43 (1972) 2235.

[38] J. Pascual, F. Peris, T. Boronat, O. Fenollar, R. Balart, Study of the effects of multiwalled carbon nanotubes on mechanical performance and thermal stability of polypropylene, Polym. Engin. Sci 52 (2012) 733-740.

[39] C. W. M. Bastiaansen, P. J. R. Leblans, P. Smith, The Theoretical Modulus of Biaxially Oriented Polymer-Films, Macromolecules 23 (1990) 2365-2370.

[40] J. Yu, H. K. Choi, H.S. Kim, S.Y. Kim, Synergistic effect of hybrid graphene nanoplatelet and multi-walled carbon nanotube fillers on the thermal conductivity of polymer composites and theoretical modeling of the synergistic effect, Composites Part A 88 (2016) 79-85.

[41] J.N. Coleman, U. Khan, W.J. Blau, Y.K. Gun'ko, Small but strong: a review of the mechanical properties of carbon nanotube-polymer composites, Carbon 44 ( 2006) $1624-1652$

[42] Q.P. Feng, X.J. Shen, J.P. Yang, S.Y. Fu, Y.W. Mai, K. Friedrich, Synthesis of epoxy composites with high carbon nanotube loading and effects of tubular and wavy morphology on composite strength and modulus, Polymer 52 (2011) 6037-6045. 
[43] R. Arasteh, M. Omidi, A.H.A. Rousta, H. Kazerooni, A study on effect of waviness on mechanical properties of multi-walled carbon nanotube/epoxy composites using modified Halpin-Tsai theory, J. Macromol. Sci. Part B Phys. 50 (2011) 2464-2480.

[44] M.K. Yeh, N.H. Tai, J.H. Liu, Mechanical behavior of phenolic-based composites reinforced with multi-walled carbon nanotubes, Carbon 44 (2006) 1-9.

[45] Y. Brechet, J.Y. Cavaillé, E Chabert, L. Chazeau, R. Dendievel, L. Flandin, C. Gauthier, Polymer based nanocomposites : effects of filler-filler and filler-matrix interactions, Adv. Eng. Mat. 3 (2001) 571-577.

[46] V. Favier, H. Chanzy, J.Y. Cavaillé, Polymer nanocomposites reinforced by cellulose whiskers, Macromol. 28 (1995) 6365-6367.

[47] S. Etienne, J.Y. Cavaillé, J. Perez, R. Point ,M. Salvia, Automatic system for analysis of micromechanical properties, Rev. Sci. Instrum. 53 (1982) 1261. 